\title{
Modelización matemática desde la perspectiva contextualizada
} Mathematical modeling from the contextualized perspective.

\section{A modelação matemática a partir da perspectiva contextualizada.}

\author{
Juan Luquez Herazo' \\ Juan Pacheco Fernández ${ }^{2}$ \\ Ever De La Hoz Molinares ${ }^{3}$ \\ Universidad Popular del Cesar
}

\section{RESUMEN}

En el artículo se presentó una revisión del concepto y el proceso de modelización, desde la perspectiva de las escuelas de educación matemáticas contextualizadas. La investigación

1 jenriqueluquez@unicesar.edu.co $\quad$ https://orcid. org/0000-0001-9815-3942 Egresado de Licenciatura en Matemáticas y Física, Universidad Popular del Cesar, Valledupar, Cesar, Colombia.

2 juanpacheco@unicesar.edu.co https://orcid.org/00000002-1333-5040 Profesor. Departamento de Física. Facultad Ciencias Básicas y Educación, Universidad Popular del Cesar, Valledupar, Cesar, Colombia.

3 everdelahoz@unicesar.edu.co $\underline{\text { https://orcid. }}$ org/0000-0003-2015-9054 Profesor. Departamento de Matemáticas y Estadística. Universidad del Cesar, Valledupar, Colombia. fue racionalista de análisis de tipo documental, complementada con el sustento empírico de avances de las investigaciones realizadas por los autores sobre la enseñanza de la matemática escolares a partir de situaciones contextualizadas.

\section{PALABRAS CLAVE}

Situaciones contextualizadas, educación matemática, matemáticas escolares.

\section{ABSTRACT}

The article presents a review of the concept and the modeling process, from the perspective of contextualized schools of mathematics 
education. The research is rationalist analysis of documentary type, complemented with the empirical support of advances of the investigations carried out by the authors on the teaching of school mathematics from contextualized situations.

\section{KEY WORDS:}

Contextualized situations, mathematics education, school mathematics

\section{RESUMO}

O artigo apresentou uma revisão do conceito e do processo de modelação, a partir da perspectiva das escolas contextualizadas de educação matemática. A pesquisa foi uma análise racionalista de tipo documental, complementada com o suporte empírico dos avanços das investigações realizadas pelos autores sobre o ensino da matemática escolar a partir de situações contextualizadas.

\section{PALAVRAS-CHAVE:}

Situações contextualizadas, educação matemática, matemática escolar

\section{INTRODUCCIÓN}

Este artículo presenta cómo algunos autores conceptualizan la modelización en el aula desde las escuelas de educación matemática y tendencias contextualizadas, para esto se consultó cómo se aborda el concepto de modelización desde las diferentes investigaciones en educación matemática.

Se presenta cómo se asume desde cada estudio la modelización matemática, luego se analizan las diferentes estrategias didácticas seguidas en las investigaciones durante el proceso de modelización matemática que realizan los estudiantes de acuerdo a las situaciones contextualizadas propuestas.
En este orden de ideas surgen ciertos interrogantes, ¿Qué concepción existe acerca de la modelización matemática? La modelización matemática es una tarea compleja, en la cual el rol del docente es el mediador entre el conocimiento y el estudiante en el proceso enseñanza-aprendizaje de las matemáticas escolares. Esto lo hace mediante el diseño e implementación de situaciones contextualizadas donde el estudiante mediante la interpretación, argumentación de estas desarrolle las competencias básicas en matemáticas de (razonamiento, resolución de las situaciones a través de un modelo matemático propuesto y comunicación). (Blomhøj, 2008).

La modelización matemática como estrategia de enseñanza aprendizaje de las matemáticas escolares juega un papel fundamental, porque permite al docente considerar el contexto físico y social para desarrollar actividades con situaciones problema en contextos vinculados con el día a día de los estudiantes, así como favorecer "la comprensión de conceptos matemáticos".

¿Cómo interviene la contextualización? esta interviene en la descripción, interpretación y análisis de una situación problema del contexto social o situación de la vida cotidiana que le permiten a los estudiantes trabajar con actividades, fenómenos de la vida real familiares a ellos y de acuerdo a sus intereses, haciendo uso de sus conocimientos matemáticos durante la modelización, lo que se considera crucial en el proceso enseñanza-aprendizaje de las matemáticas escolares; debido a que permite pasar de la enseñanza tradicional (rol pasivo del estudiante al activo), donde el docente es el poseedor del conocimiento a tener un papel de mediador entre el conocimiento y el estudiante; este pasa hacer parte activa debido a que cambia de receptor a ser investigador durante la modelización de la actividad propuesta (Camelo, Perilla, \& Mancera, 2016). 
El proceso de modelización conduce a un acceso democrático e inmediato del conocimiento por parte del estudiante y a la aprehensión del mismo; además, conlleva que el estudiante lo coloque en juego en la resolución de las situaciones contextualizadas propuestas de su entorno, esto le permite establecer que los saberes escolares adquiridos son herramientas fundamentales en la interpretación, análisis, resolución de estas a través de la modelización matemática (Zamora, 2013), mostrándole la importancia y necesidad de estas en el proceso enseñanza-aprendizaje de las matemáticas escolares; además, de servir de estrategia de motivación, porque existe una transformación de un rol pasivo a un rol activo, en cual el docente se convierte en un orientador del proceso, lo que le permite al estudiante ver la importancia, utilidad y lo imprescindible de las matemáticas no solo para los matemáticos, sino para toda la sociedad. (Biembengut \& Hein, 2004)

Las escuelas de educación matemáticas que consideran a la matemática como una actividad ligada al contexto social, siendo coherente con sus concepciones epistemológicas que consideran que en el proceso de enseñanzaaprendizaje de las matemáticas escolares es esencial partir de las actividades que realizan los estudiantes en su contexto social. Por ende, las escuelas que cumplen estas características se denominan escuelas de educación matemática contextualizadas. En este estudio, se analiza el concepto y procedimientos de modelización matemática seguidas por algunas de las principales escuelas de esta perspectiva, entre las que se puede mencionar: Educación matemática crítica, educación matemática realista, Socio epistemológica y teoría antropológica de lo didáctico, TAD.

\subsection{Modelización matemática a partir del contexto}

La modelización en las diferentes escuelas de educación matemática contextualizadas se fundamenta en situaciones o fenómenos provenientes de la realidad (Blum \& Borromeo, Mathematical Modelling: Can It Be Taught and Learnt?, 2009).

Este proceso que traslada el mundo real a las matemáticas en donde el factor influyente es el docente, dado que tiene que ubicar en contexto una situación, en la cual los estudiantes puedan trabajar con un fenómeno o situación de la vida diaria que les sean familiar y que les permita situar en juego su conocimiento matemático en un proceso de modelización. El espacio para determinar las actividades contextualizadas para la modelización, lo determina el contexto social.

El contexto social, puede ser comprendido como un camino para el proceso de la enseñanza y el aprendizaje de las matemáticas, partiendo de la realidad o del contexto de los estudiantes. De esta manera, el estudiante pasa a ser corresponsable de su aprendizaje, y el rol del maestro es ser un orientador despertando el sentido crítico y creativo, logrando transformar problemas de la realidad en problemas matemáticos para luego ser resueltos (Biembengut \& Hein, 2004)

De este modo la modelización puede ser considerada como un dispositivo didáctico de representación de situaciones o fenómenos del mundo real. La modelación es entonces una estrategia que posibilita el entendimiento de un concepto matemático inmerso en un contexto dotado de relaciones y significados que prepara al estudiante para ir desarrollando una actitud diferente y abordar los problemas de un contexto real (Villa, 2007).

El proceso de modelización matemática se basa en la identificación con el mundo real: Análisis del problema, es por lo tanto comprender los requerimientos del mundo real y sus restricciones en ese contexto. La matematización, involucra la formulación de la situación real y la construcción del modelo. La Solución del modelo, es a través 
de la manipulación matemática. La Validación del modelo, es cuando se da una predicción confiable del comportamiento del sistema y, por último, la implementación del resultado matemático final en el sistema real (King, Garrett, \& Coghill, 2005)

El proceso de modelización cumple con su cometido fundamental de conseguir que el estudiante aprenda. De este modo, el equilibrio entre la necesaria autonomía del alumno y la necesaria intervención del profesor provoca en el profesor dilemas y tensiones, motivadas porque la enseñanza se convierte en más abierta y menos predecible, (Blum \& Borromeo, Mathematical Modelling: Can It Be Taught and Learnt?, 2009).

\section{METODOLOGÍA}

La investigación fue de carácter racionalista, consistió en realizar una revisión y un análisis documental de los artículos científicos publicados en idioma español en revistas indexadas por los principales representantes de las escuelas de educación matemática contextualizadas; se usó el criterio de contextualización mencionado al final de la introducción de este artículo.

El corpus del estudio fueron los artículos que cumplieron con la característica de la modelización en las escuelas de educación matemática desde una perspectiva contextualizada, donde las fuentes de consulta para conformar el corpus fueron: los artículos revisados en la línea de investigación de educación matemática del grupo de investigación educativa en Ciencias Naturales y Matemática, (ECINAMA) de la Universidad Popular del Cesar (UPC), para realizar sus proyectos de investigación, complementada con una consulta en el buscador Google Académico utilizando como frases claves: modelización, modelización matemática, situaciones contextualizadas, modelización matemática desde una perspectiva critica, modelización matemática desde una perspectiva realista, modelización matemática desde una perspectiva socio epistemológica y teoría antropológico de lo didáctico.

\subsection{ANÁLISIS DOCUMENTAL DE LA MODELIZACIÓN MATEMÁTICA.}

En los artículos seleccionados como corpus, se analizó el concepto de modelización en las escuelas de educación matemática contextualizadas, de acuerdo con lo expuesto en la introducción de este artículo, así:

$1^{\circ}$. Con la finalidad de tener en cuenta los elementos y aspectos comunes de cada uno de los marcos teóricos, desde las perspectivas de algunos autores de cada de ellas.

$2^{\circ}$. Se realizó el análisis de los diferentes pasos propuestos por cada uno de los autores consultados de las escuelas contextualizadas, que son realizados por el estudiante en el proceso de modelización matemática de la situación asociada al contexto propuesta en clase.

$3^{\circ}$. Las escuelas de educación matemática en las que se analizó la modelización matemática desde una perspectiva contextualizada son: la educación matemática crítica, realista, la Socio epistemológica y la Teoría Antropológico de lo Didáctico (TAD).

Los resultados de este análisis y una aproximación a los lineamientos para la modelización matemática desde una perspectiva contextualizada se presentan en la siguiente sesión, lo didáctico.

\section{RESULTADOS Y DISCUSIÓN.}

Realizada la revisión bibliográfica de los artículos científicos publicados en cada una de las escuelas contextualizadas se presentan los resultados del análisis desde cada una de ellas, se proponen los pasos que debe seguir el 
estudiante en el proceso de modelización de la situación contextualizada propuesta.

Los resultados que se obtuvieron son los siguientes:

\subsection{LA MODELACIÓN MATEMÁTICA DESDE LA PERSPECTIVA CRÍTICA}

La educación matemática crítica busca descubrir y transformar las relaciones de poder que están implícitas en las actividades matemáticas desarrolladas en las clases, esto conlleva a que los profesores y estudiantes se relacionen en una situación de aprendizaje y enseñanza que tengan como propósito el desarrollo de competencias democráticas que tengan como propósito fundamental la práctica y la teoría del conocimiento reflexivo en matemáticas, la comunicación, la reflexión, y el diálogo, parallevar a cabo la modelización matemática crítica según (Jacobini, 2004), son vistos los posicionamientos críticos y las posturas democráticas, como aquellos elementos útiles para que el estudiante transforme su sociedad. De modo que, además de adquirir el conocimiento, el estudiante adquiere las habilidades necesarias para usar estos conocimientos; es decir, ofrece la oportunidad según (Valero, 2002) que se represente, analice, modele y tome decisiones respecto a una situación de su cotidianidad.

En este sentido, la Modelación Matemática desde la perspectiva crítica, permite vincular el contexto socio-cultural en el cual están inmersos los estudiantes con la matemática, permitiéndole esto, el desarrollo de competencias democráticas necesarias para ser actores responsables en su sociedad, (Valero, 2002)

La modelación matemática resulta determinante en la integración de los modelos matemáticos y de la modelación matemática, como medios de aprendizaje de las matemáticas. Razón por la cual se puede deducir que la modelación matemática, desde una mirada educativa, busca adaptar o recrear las etapas de la actividad científica en la escuela para la comprensión o construcción de un concepto matemático.

La modelación matemática, está orientada por aspectos sociales, políticos y críticos, los cuales promueven la participación crítica de los estudiantes/ciudadanos en la sociedad, al discutir asuntos políticos, económicos o ambientales en los que las matemáticas sirven como soporte tecnológico (Skovsmose, 1994).

Tal y como han planteado (Mancera, Camelo, Salazar, \& Valero, 2012), los contextos socialmente relevantes de los estudiantes, elegidas colectivamente por ellos o propuestas por los profesores para que, como marco, sean usados por los estudiantes para plantear problemáticas y preguntas específicas para abordar y reflexionar (Barbosa J. , 2003), (Camelo, Perilla, \& Mancera, 2016) y (Salazar, Mancera, Camelo, \& Perilla, 2017).

En consecuencia, (Araújo, 2009), asume que los modelos matemáticos posibilitan abrir espacios donde es posible que se aporte a la constitución del pensamiento crítico de los estudiantes, por lo que se da énfasis a la integración de escenarios donde los niños, niñas y adolescentes puedan actuar críticamente frente a las problemáticas que se deciden abordar, soportados en comprensiones matemáticas que van construyendo colectivamente con sus compañeros.

Los ambientes de aprendizaje propuestos por (Skovsmose, 2000) permiten visualizar las diversas maneras en que se trabaja en el aula, al combinar las formas de organizar la actividad de los estudiantes con el tipo de referencia, ambientes que, para el enfoque de la Educación Matemática Crítica, EMC, van desde lo considerado como "tradicional", hasta el ambiente tipo considerado como "ideal". 


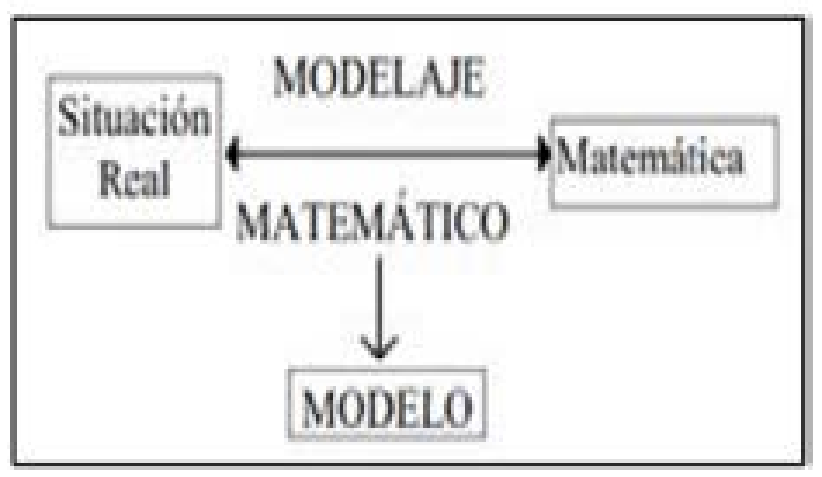

Figura 1. Esquema del proceso de modelaje matemático

Fuente:https://revistas.udistrital.edu.co/index. php/revcie/article/download/7054/8726/

La perspectiva crítica a través del esquema anterior permite conectar situaciones del entorno de los estudiantes, incorporando aspectos democráticos, que se fundamenta en la posibilidad de crear un lenguaje con nuevas visiones, sobre las matemáticas escolares y el desarrollo de una ciudadanía crítica.

La educación matemática crítica, permite a los ciudadanos ejercer las competencias democráticas, y el poder formativo de las matemáticas para tomar posiciones críticas, a través del conocer reflexivo mediante el modelaje matemático. (Skovsmose, 1999).

Otros autores la consideran como un enfoque sociocrítico la cual, considera el desarrollo de ambientes de modelación matemática en el aula de clase, desde la perspectiva sociocrítica, donde promueve, tal y como señala (Blomhoj, 2009), (Kaiser \& Sriraman, 2006), (Barbosa J. , 2009) (Araújo, 2009), participaciones críticas de los estudiantes, gracias a las reflexiones sobre los modelos matemáticos que están construyendo, situación que permite empoderarlos como ciudadanos autónomos e independientes en la sociedad.
En este sentido (Rojas, Salazar, \& Romero, 2013); (Blomhoj, 2009); (Mancera, Camelo, Amaya, \& García, 2014); y (Barbosa J. , Modelagem Matemática: $O$ que é? Por que? Como?, 2004), resaltan que los ambientes de modelación matemática en la perspectiva sociocrítica pueden comenzarse de tres maneras diferentes a saber:

i) El profesor propone a los estudiantes sobre qué investigar, delimitando el problema claramente.

ii) El profesor coloca un marco general en el que los estudiantes pueden decidir sobre qué investigar.

iii) Los estudiantes proponen sobre quién investigar.

Los aspectos que están presentes en el aprendizaje de las matemáticas critica se reflexiona sobre cada uno de los asuntos que hacen parte el salón de clase. Esto hace que las matemáticas tomen un lenguaje poderoso, que permite producir nuevos proyectos dentro del entorno de la actividad en estudio.

Por lo cual se presentan algunas investigaciones en educación matemática soportado desde el marco teórico de esta escuela, en las cuales se resaltan el propósito y los resultados de cada investigación.

1. "propuesta de ambientes de aprendizaje para la promoción de la modelación matemática desde la perspectiva crítica", la investigación hizo uso de la metodología investigación-acción y, desarrollo de una propuesta de enseñanza-aprendizaje para visualizar los procesos de Modelación Matemática usados por estudiantes de secundaria de un colegio Distrital de Bogotá al abordar Ambientes de Aprendizaje desde la propuesta de Skovsmose (Bustos, Bustos, \& Novoa, 2013). 
2. matemática desde una perspectiva crítica" en esta investigación el ambiente de clase posibilitó que los y las jóvenes trabajaran en grupos y evidenciaran posicionamientos críticos, constituyéndose, junto con aspectos políticos y sociales, en posibilidades para que los estudiantes cuestionaran la realidad. Como desafíos se estableció que la participación activa de los y las jóvenes en su contexto, es puntualizar profundamente las reflexiones analíticas argumentativas de los análisis del trabajo en cuestión. (Perilla, Mancera, \& Camelo, 2016).

3. Prácticas de modelación matemática desde una perspectiva socio crítica con estudiantes de grado undécimo, se basa en la creación y análisis de un ambiente de modelación matemática desde la perspectiva socio-crítica (Araújo, 2009), desarrollado con un grupo de estudiantes de grado undécimo en un colegio público de Bogotá, Colombia. Con objetivo central de evidenciar posibilidades y desafíos al incorporar i) problemas socialmente relevantes, ii) participación activa de los estudiantes en la construcción del modelo, iii) participación activa de los estudiantes en la sociedad, iv) actuación del profesor como mediador. (Perilla, Mancera, \& Camelo, 2016)

\subsection{LA MODELIZACIÓN MATEMÁTICA DESDE LA PERSPECTIVA REALISTA}

Este enfoque teórico se basa en unos principios de enseñanza y aprendizaje, cuyas directrices para la enseñanza de las matemáticas surgen como consecuencia natural de las ideas alcanzadas sobre el aprendizaje de las matemáticas. (Goffree, 2000), su idea central es que los estudiantes aprenden matemáticas desarrollando y aplicando conceptos y herramientas en situaciones de la vida diaria, el trabajo con contextos que promuevan la producción y usos de modelos matemáticos; es más productivo cuando el objeto matemático central interviene en la situación-problema.
Elenfoque de modelización de laEMRseenmarca fundamentalmente dentro del principio de niveles que se refiere al proceso de matematización que va sucediendo en una progresión, no estricta, de micro niveles, lo que (Treffers, 1987), denomina: matematización progresiva. Los objetivos que tiene la modelización matemática, es promover el desarrollo de la teoría; es decir, lograr que los contenidos fundamentales de la matemática sean aprendidos en el trabajo con la modelación de fenómenos reales, pero sin perder aspectos importantes de la epistemología de los conceptos.

El proceso de modelación, se evalúa a través de la validación de datos reales y, en consecuencia, es necesario estudiar con profundidad la modelación en diferentes profesiones y áreas de aplicación que den lugar a la construcción de modelos matemáticos. (Blomhoj, 2009)

En consecuencia, los contextos tomados del mundo real son comprensibles para los estudiantes y la modelación como tal debería estar en el horizonte para ellos. En esta perspectiva se puede ubicar el enfoque de la conceptualización desarrollado por (Biembengut \& Hein, 2004), quienes entienden la modelación como un proceso que tiene como objeto la obtención de un modelo matemático de un fenómeno o una situación problema. 
Tabla 1. Modelación matemática desde la perspectiva realista

\begin{tabular}{|c|c|}
\hline \multicolumn{2}{|r|}{ PROCESO DE MODELACIÓN MATEMÁTICA DESDE LA PERSPECTIVA REALISTA } \\
\hline $\begin{array}{l}\text { Principio } \\
\text { De } \\
\text { actividad }\end{array}$ & $\begin{array}{l}\text { Los estudiantes se enfrentan a situaciones problemas en las cuales ellos mismos a través } \\
\text { de sus conocimientos informales "reinventan" las matemáticas como participantes } \\
\text { activos durante el proceso de aprendizaje. (Panhuizen, 2008). } \\
\text { El proceso de modelación matemática se denomina matematización y puede estudiarse } \\
\text { a partir de dos niveles: } \\
\text { La matematización horizontal entendida como el proceso mediante el cual los } \\
\text { estudiantes logran trasladar el problema de su contexto a algún tipo de matemáticas, } \\
\text { mediante métodos informales o prefórmales a diferentes niveles de abstracción (Arcavi, } \\
\text { 2006). } \\
\text { La matematización vertical puede entenderse como el proceso que lleva a la elevación } \\
\text { del pensamiento matemático abstracto, puesto que en este nivel la organización } \\
\text { matemática se realiza dentro del mismo sistema matemático sin referenciar el contexto } \\
\text { del cual se desprende la situación problema. (Fernández \& Velázquez, 2007). }\end{array}$ \\
\hline $\begin{array}{l}\text { Principio } \\
\text { De } \\
\text { realidad }\end{array}$ & $\begin{array}{l}\text { Parte de contextos y situaciones realistas con el ánimo que los estudiantes sientan la } \\
\text { necesidad de matematizar la situación problema. Dichos contextos y sus situaciones } \\
\text { realistas guardan alguna conexión con el mundo real, pero son ante todo situaciones } \\
\text { que son reales en la mente de los estudiantes, y por tanto las situaciones realistas } \\
\text { tienen un carácter relativo que depende exclusivamente de la experiencia previa de los } \\
\text { estudiantes y/o de la capacidad de estos para imaginar la situación y no necesariamente } \\
\text { implica que los problemas provienen del mundo real. } \\
\text { De tal manera, que las situaciones limitarían las posibilidades de los estudiantes para } \\
\text { aprender a operar dentro de los sistemas matemáticos. (Bressan \& Gallego, 2011) }\end{array}$ \\
\hline $\begin{array}{l}\text { Principio } \\
\text { De } \\
\text { nivel }\end{array}$ & $\begin{array}{l}\text { Durante el proceso de modelación matemática los estudiantes pasan por diferentes } \\
\text { niveles de comprensión: desde la capacidad para inventar soluciones informales } \\
\text { estrechamente ligadas al contexto pasando por esquematizaciones generales de la } \\
\text { situación, hasta llegar a la adquisición de relaciones más amplias aplicables a otros } \\
\text { contextos y situaciones (Bressan \& Gallego, 2011), (Panhuizen, 2008) }\end{array}$ \\
\hline $\begin{array}{l}\text { Principio } \\
\text { De } \\
\text { entrelazamiento }\end{array}$ & $\begin{array}{l}\text { Esta tiene una fuerte interrelación e integración entre los contenidos matemáticos } \\
\text { escolares, puesto que la resolución de situaciones realistas a menudo exige establecer } \\
\text { conexiones con una amplia variedad de herramientas y conocimientos matemáticos. } \\
\text { Así pues, (Bressan \& Gallego, 2011) afirman que este enfoque no hace mayores } \\
\text { distinciones entre las unidades curriculares, generando coherencia a la enseñanza y } \\
\text { facilitando que se den modos de matematizar muy diferentes. }\end{array}$ \\
\hline
\end{tabular}




\begin{tabular}{|l|l|}
\hline $\begin{array}{l}\text { Principio } \\
\text { De }\end{array}$ & $\begin{array}{l}\text { El aprendizaje de las matemáticas es una actividad social donde la interacción colectiva } \\
\text { (estudiante-estudiante/s y estudiante-docente), fortalece la elevación en los niveles de } \\
\text { comprensión. Esto no implica que todos los estudiantes alcanzan el mismo nivel de } \\
\text { comprensión, sino que cada estudiante sigue su trayectoria propia de aprendizaje. }\end{array}$ \\
$\begin{array}{l}\text { Además, es esencial que el docente encuentre el momento oportuno para incluir } \\
\text { la reflexión en el salón de clases y que anticipe cuando la interacción social puede } \\
\text { obstaculizar el proceso de aprendizaje (Goffree, 2000). }\end{array}$ \\
$\begin{array}{l}\text { Principio } \\
\text { orientación }\end{array}$ & $\begin{array}{l}\text { Los docentes desempeñan un papel crucial en la forma cómo los estudiantes adquieren } \\
\text { conocimientos, y es indispensable que estos promuevan espacios a través de los cuales } \\
\text { se puedan construir los saberes matemáticos. } \\
\text { De ninguna forma el docente debe olvidar que es un mediador entre las producciones } \\
\text { informales de sus estudiantes y las estructuras formales de la matemática }\end{array}$ \\
\hline
\end{tabular}

Fuente: https://core.ac.uk/download/pdf/19100132.pdf

La Educación Matemática Realista, ofrece un marco teórico y metodológico que podría ser fructífero para promover un acercamiento particular a los procesos de modelación matemática en el aula y materializarse en estrategias de intervención en las aulas de matemáticas.

Desde esta perspectiva realista se encuentran algunas investigaciones:

1. “Educación matemática realista: la modelación matemática en la producción y uso de modelos cuadráticos", en esta investigación se destaca el papel que desempeña el proceso de modelación matemática en la conjugación de las matemáticas y la realidad para la promoción de la formación de conceptos matemáticos asociados a lo cuadrático, donde se asumen los diversos niveles de matematización, como una posibilidad que permite analizar el desempeño matemático de los estudiantes y las implicaciones didácticas y cognitivas, en relación con el proceso de modelación en el ámbito escolar (Vanegas \& Henao, 2013)

2. Modelización matemática en la educación matemática realista: Una propuesta para contribuir a la construcción formal de álgebra lineal, el objetivo que se plantío en esta investigación fue diseñar, implementar y evaluar una secuencia didáctica basada en el uso de la modelización matemática en la EMR que promueva la construcción de conjunto generador y espacio generado. (Cárcamo, Gómez, \& Fortuny, 2015)

\subsection{La modelización matemática desde la perspectiva socio epistemológica}

La Teoría Socio epistemológica de la Matemática Educativa (Cantoral, Teoría socioepistemológica de la matemática educativa. Estudios sobre construcción social del conocimiento (1a ed., 2013), plantea la práctica social como un constructo teórico central con diferentes funciones, entre ellas la normativa, la discursiva, la identitaria y la pragmática. Reconocen la diferencia epistemológica entre práctica social y 
práctica situada. Si bien se abordan cuestiones pragmáticas, discursivas y normativas, ello se emprende desde la observación directa en la práctica social.

(Cantoral, Montiel, \& Reyes-Gasperini, 2015) La Socioespistemología promueve una muy particular forma de estudiar los procesos de enseñanza y de aprendizaje de las Matemáticas: abandona una tradicional mirada centrada en objetos hacia otra centrada en prácticas que es guiada por el constructo teórico de práctica social. Esto significó en su momento un cambio fundamental en la manera en que suele investigarse en Matemática Educativa, donde la enseñanza empieza con el objeto matemático explícito u ostensible. En nuestro caso, se estudiará las prácticas asociadas al objeto.

La modelación desde el punto de vista Socio epistemológica está basada como una construcción teórica (Cordero, 2006) que un individuo realiza al enfrentar una tarea matemática en la que pone en juego sus conocimientos. $Y$ como características propias de esta construcción, la modelación posee su propia estructura, está constituida por un sistema dinámico, la simulación puede llevar a cabo realizaciones múltiples y hacer ajustes en su estructura para producir un resultado deseable, es un medio que propicia el desarrollo del razonamiento y de la argumentación, busca explicaciones a un rango y enfatiza invariantes, trae una idea en una realización para satisfacer un conjunto de condiciones. Con todas estas características, la modelación selecciona el lenguaje de las herramientas (Arrieta, 2003) sobre el lenguaje de los objetos.

La Teoría Socio-epistemológica (TS) trata con fenómenos de producción, adquisición y de difusión del conocimiento matemático desde una perspectiva sistémica y múltiple, cuya finalidad es el rediseño del discurso Matemático Escolar. Para ello, descentra la mirada en los objetos y problematiza al saber, al plantear la incorporación a la investigación de la epistemología del conocimiento, de su dimensión sociocultural, los procesos cognitivos asociados y los mecanismos de institucionalización vía su enseñanza (Cantoral, Teoría socioepistemológica de la matemática educativa. Estudios sobre construcción social del conocimiento (1a ed., 2013).

La mirada sobre lo que se produce en la práctica de modelación y su función, cuando los objetos matemáticos no son el centro de la discusión o cuando el énfasis no se encuentra en el análisis de los procesos cognitivos que acompañan a las tareas de modelación. Pero, por otro lado, este posicionamiento implica un viraje en el diseño de actividades, puesto que se ingresan categorías de conocimiento matemático que se plantean como socio epistemológicas, que devinieron de prácticas sociales y se habilitan en lo escolar.

El esquema metodológico para la investigación Socio epistemológica (Espinosa \& Buendía, 2012). Dicho esquema, está construido en base a las investigaciones realizadas por (Buendía, 2013) y (Montiel, 2011), el cual ha sido retroalimentado por diferentes investigaciones junto con diversas discusiones dentro de la comunidad científica. 
Figura 2. Esquema metodológico socio epistemológico

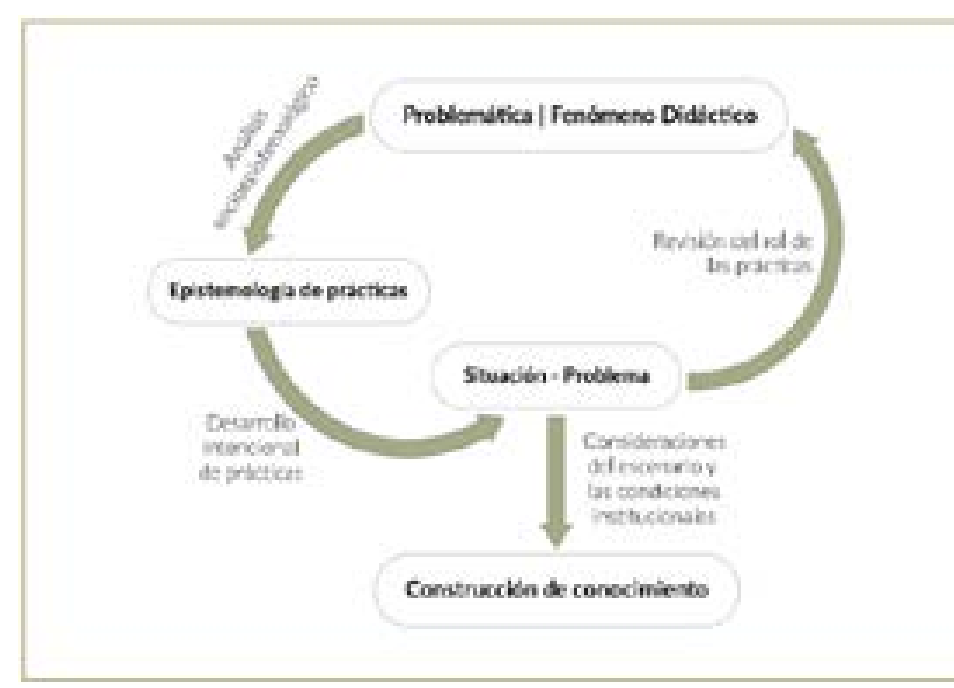

Fuente:http://revistaucmaule.ucm.cl/article/ download $/ 462 / 634$ ? inline $=1$

En términos estructurales, (Montiel \& Buendía, Un esquema metodológico para la investigación socioepistemológica: ejemplos e ilustraciones, 2013)está compuesto de nodos, los cuales se conforman de un conjunto de tareas propias. Dichos nodos están, a su vez, unidos por medio de acciones relacionantes (representadas por flechas). Es importante señalar que una investigación que utilice este tipo de esquema metodológico no necesariamente debe abarcar la totalidad de nodos; dependiendo de los propósitos de investigación, puede estar compuesta de uno sólo o una combinación de estos, unidos por medio de ciertas acciones relacionantes.

Desde un enfoque Socio epistemológico, la matemática no es concebida como un conocimiento ajeno a la realidad del que aprende. Por ello, al plantear la categoría de modelación, existe una necesidad por crear una variedad teórica en cuanto al principio que concilia el enfoque tradicional. Dicha variedad se ha ido construyendo y robusteciendo a partir de lo realizado en diferentes investigaciones socio epistemológicas (Cordero, 2017) (Arcos, 2017) y (Codero, Mena-Lorca, Huincahue, Mendoza, \& Pérez-Oxté, 2019), en donde se considera una variación del principio $\mathrm{P}$, que considera la funcionalidad del conocimiento matemático en la práctica educativa.

Desde esta perspectiva realista se encuentran algunas investigaciones:

1. La graficación - modelación y la Serie de Taylor. Una Socioespistemología del cálculo, la investigación está basada en la resignificación de la Serie de Taylor en una situación de modelación del movimiento. De acuerdo con la perspectiva epistemológica. Los ejes principales de la situación son la predicción y el binomio graficación-modelación, en cuanto prácticas sociales. Estos articulados generan conocimiento y resignifican la Serie de Taylor. (Morales \& Cordero, 2014)

\section{2. la categoría de modelación y} el concepto de integral definida: una mirada Socio epistemológica, la presente investigación se basó en desarrollar la categoría de modelación como una resignificación de usos de la acumulación en dos situaciones específicas de variación, una perteneciente al dominio de la Fenología y la otra al dominio de la Economía. Dicho desarrollo permite dar bases para el diseño de situaciones escolares de socialización en educación superior cuya finalidad sea incorporar dichos usos a los procesos de construcción del concepto de integral definida, considerando la funcionalidad del conocimiento matemático, la pluralidad epistemológica y la transversalidad del saber. (PERALTA, 2020)

3. Modelación - graficación, una categoría para la matemática escolar. Resultados de un estudio socio epistemológico, en esta investigación se plantean hipótesis sobre la naturaleza de la construcción social de conocimiento del Cálculo asociado a la variación y el cambio. El resultado de esta investigación es el planteamiento de 
una epistemología para la modelación escolar caracterizada a través de un uso de las gráficas. (Tellez \& Cordero, 2010)

\subsection{LA TEORÍA ANTROPOLÓGICA DE LO DIDÁCTICO}

La Teoría Antropológica de lo Didáctico (en adelante, TAD) aparece con las primeras formulaciones de la Teoría de la Transposición Didáctica (Chevallard \& Johsua, La transposition didactique: du savoir savant au savoir enseigné, 1985).Puede ser considerada como un desarrollo de la Teoría de las Situaciones Didácticas (Brousseau, (1997) con la que comparte sus principios fundamentales. Los procesos de estudio de esta consisten en la construcción y reconstrucción de praxeologías de complejidad creciente. Veamos qué son las praxeologías. Desde la TAD se postula que cualquier actividad humana, en particular el estudio de la matemática, se puede modelar en términos de una componente práctica (praxis) y una componente teórica (logos). La praxis corresponde al saber hacer y se compone del tipo de tareas y las técnicas o modos de resolver las tareas. El logos corresponde al saber y se compone de la tecnología y la teoría. (Rey, 2017) La TAD, como marco teórico, parte de una concepción de la didáctica de las matemáticas como la ciencia que estudia la difusión y a adquisición del conocimiento matemático (Chevallard \& Sensevy, 2014).

La modelización desde el punto de vista de las TAD se ha entendido como una respuesta matemática a problemas de contexto extramatemático, mediante la aplicación del ciclo de modelización (Blum \& Niss, 1991).Para introducir al alumno en un proceso de modelización que le permita la adquisición del conocimiento matemático pretendido, o para adquirir la "capacidad" de modelizar, la modelización no es sólo una dimensión de la actividad matemática, sino que la actividad matemática es, en esencia, una actividad de modelización

Los procesos de modelización pueden desempeñarse en la enseñanza y el aprendizaje de las matemáticas en todos los niveles del sistema educativo. Durante mucho tiempo, la "modelización" ha estado restringida a la aplicación de un conocimiento matemático previamente construido a determinadas situaciones "reales". Hoy día, perdura este uso del término "modelización".

Figura 3. Ciclo de modelización

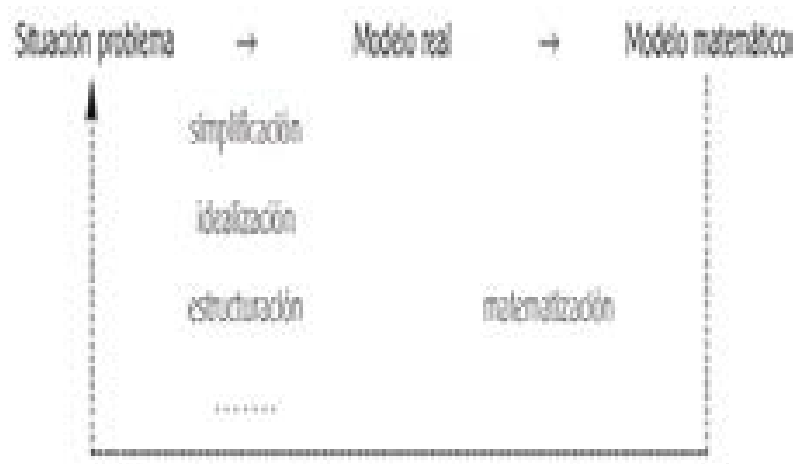

Fuente: https://www.redalyc.org/ pdf/405/40558507003.pdf

La propuesta por (Blum \& Niss, 1991), continúa y produce, basándose en la experimentación y en la reflexión (metodología muy próxima a la de la investigación-acción), los procesos de modelización se producen desde dos ámbitos diferentes, consisten en desarrollar un proceso de simplificación, idealización y estructuración de una situación problema para formular un modelo real. A partir de este, mediante un proceso de matematización, se construye un modelo matemático. El ciclo se cierra al devolver a la situación problema el modelo matemático construido para su contrastación.

La TAD se sitúa dentro del Programa Epistemológico y postula que "gran parte de la actividad matemática puede identificarse (...) 
con una actividad de modelización matemática" (Chevallard, Bosch, \& Gascón, 1997) la modelización no es sólo una dimensión de la actividad matemática, sino que la actividad matemática es, en esencia, una actividad de modelización.

La investigación actual en modelización y aplicaciones centrada en la enseñanza explícita de la modelización, implica una necesaria problematización de los procesos de modelización matemática en un intento de superar la ilusión de transparencia implícita en gran parte de las investigaciones. Esta problematización de los procesos de modelización se produce desde dos ámbitos (Bosch, García, Gascón, \& Higueras, 2006)

Tabla 2. Problematización de los procesos de modelización matemática

\begin{tabular}{|l|l|}
\hline $\begin{array}{l}\text { Problematización epistemológica: necesidad de problematizar } \\
\text { las características de las "situaciones reales" que permitan el } \\
\text { desarrollo de un proceso de modelización con fines didácticos } \\
\begin{array}{l}\text { (cuestionamiento de que las “situaciones reales" posean, por sí } \\
\text { solas, propiedades didácticas). }\end{array}\end{array}$ & $\begin{array}{l}\text { Problematización cognitiva: } \\
\text { conocimiento de los procesos } \\
\text { cognitivos activados por los } \\
\text { estudiantes en la realización } \\
\text { de tareas de modelización y de } \\
\text { aplicaciones. }\end{array}$ \\
\hline
\end{tabular}

Fuente:https://www.redalyc.org/pdf/405/40558507003.pdf

La superación de esta transparencia se ha producido a través del cuestionamiento y la problematización de los procesos cognitivos activados por los sujetos en cada etapa de este ciclo, o bien en las transiciones entre etapas. En ocasiones, esto provoca el surgimiento de nuevas etapas, con el consiguiente enriquecimiento del ciclo de modelización propuesta por (Blum \& Leiss, 2005)

Figura 4. Ciclo de modelización

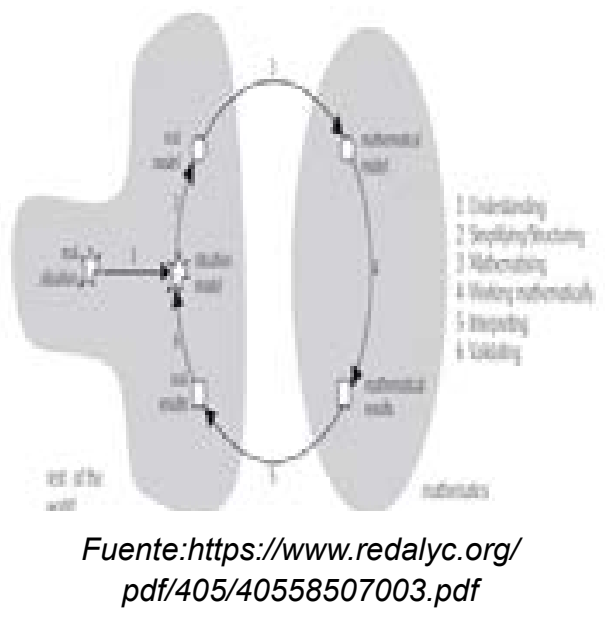

En la actualidad, en la investigación en educación matemática se considera el término "modelización" de manera más rica y fértil, configurando un amplio y heterogéneo dominio de investigación que no ha dejado de crecer en los últimos años. Tanto desde la investigación en educación matemática como desde diferentes segmentos de la sociedad, es usual oír hablar de la necesidad de relacionar los contenidos matemáticos con ciertos aspectos de la vida real y sobre la necesidad de desarrollar la "competencia modeladora" como una competencia matemática básica de cualquier ciudadano, como recoge el reciente informe mundial PISA realizado por la OCDE (Framework, 2003)

Desde esta perspectiva la Teoría Antropológica de lo Didáctico se encuentran algunas investigaciones: 


\section{El problema del álgebra elemental en la} teoría antropológica de lo didáctico propone considerar un proceso de algebrización, ampliando así el ámbito de la actividad matemática escolar en el que se suele situar el problema didáctico correspondiente. Estudiando los componentes de dicho problema, la relación entre lo numérico y lo algebraico, la algebrización de la proporcionalidad, la construcción de los números enteros como objetos algebraicos y el desarrollo de la modelización algebraica hacia la modelización funcional. (Gascón, Bosch, \& Ruiz, 2017)

\section{La introducción del álgebra elemental y su} desarrollo hacia la modelización funcional investigación parte de los trabajos en torno al problema didáctico del álgebra elemental realizados por el investigador francés Yves Chevallard. En estos trabajos se puso de manifiesto que la función principal del álgebra no es la de generalizar la aritmética sino la de modelizar sistemas intra-matemáticos o extra matemáticos. Afirmando que la enseñanza del álgebra debe promover una dialéctica entre el manejo formal del cálculo algebraico y el contenido de los sistemas numéricos. (Munzón, 2010)

\section{Modelización de situaciones de movimiento} en un sistema algebraico computacional: una aproximación desde la teoría antropológica de lo didáctico y el enfoque instrumental esta investigación combina fundamentalmente dos referentes teóricos. Una parte, la Teoría Antropológica de lo Didáctico (TAD) a partir de la cual se fundamenta la enseñanza del álgebra escolar como instrumento de modelización, la cual pretende aportar desde lo teórico y lo metodológico una visión fundamentada de la concepción del álgebra en la educación secundaria. (Pedreros, 2013)

\section{CONCLUSIONES}

Finalizada la revisión documental de algunas investigaciones, y luego de analizar los resultados de investigación obtenidos por las principales escuelas de educación matemática contextualizadas, se encuentran elementos comunes en el concepto y proceso de modelización matemática, la modelización a lo largo de la historia se ha convertido en una tarea difícil para los docentes llevar las matemáticas en contexto de los estudiantes que les permita adquirir un aprendizaje de esta misma.

Las escuelas de educación matemática contextualizadas permiten la integración de situación y actividades del contexto social en el proceso enseñanza-aprendizaje de las matemáticas escolares y que el estudiante mediante la modelización matemática proponga una respuesta óptima, a través de un modelo matemático y el lenguaje común del estudiante que le permita desarrollar todas sus competencias, habilidades y conocimiento propio.

La modelización es una herramienta de las escuelas de educación matemática contextualizadas que permite asociar eventos del entorno físico y social del estudiante y transformarla en una situación de enseñanza en el aula de clase, con la finalidad de que acceda a interpretar, describir, resolver y comunicar la situación seleccionada a través de las relaciones de sus elementos y el concepto matemático estructurante.

Este trabajo presenta una síntesis actualizada del concepto de modelización matemática y los pasos sugeridos en el proceso de modelización de la situación contextualizada presentada de las principales investigaciones de las escuelas de educación matemática contextualizadas. 


\section{REFERENCIAS BIBLIOGRÁFICAS}

Araújo, J. (2009). Uma abordagem sociocrítica da modelagem matemática: a perspectiva. Alexandria Revista de Educação em Ciências eTecnologia, Florianópolis, 55-68.

Arcavi, A. (2006). Lo cotidiano y lo académico en Matemáticas. úmeros. Revista de Didáctica de las Matemáticas, 3-23.

Arcos, J. (2017). Propuesta de modelación matemática en la formación de profesores y bases para una variedad de modelación desde la teoría Socioepistemológica. Pontificia Universidad Católica de Valparaíso.

Arrieta, J. (2003). La modelación como proceso de matematización en el aula (Tesis Doctoral).

Barbosa, J. (2003). Modelagem matemática e a perspectiva sócio-crítica. Seminário Internacional de Pesquisa em Educação Matemática, 1-13.

Barbosa, J. (2004). Modelagem Matemática: $O$ que é? Por que? Como? Veritati, 73-80.

Barbosa, J. (2009). Modelagem e Modelos Matemáticos na Educação Científica. Alexandria Revista de Educação em Ciência e Tecnologia, 69-85.

Biembengut, M. S., \& Hein, N. (2004). Modelación matemática y los desafíos para enseñar matemática. Educación Matemática, 105-125.

Blomhøj, M. (2008). Modelización matemáticauna teoría para la práctica. Revista de educación matemática.

Blomhoj, M. (2009). Different perspectives in research on the teaching and learning mathematical modelling. Mathematical applications and modelling in the teaching and learning of mathematics.

Blum, W., \& Borromeo, R. (2009). Mathematical Modelling: Can It Be Taught and Learnt? Journal of Mathematical Modelling and Application, 45-58.

Blum, W., \& Leiss, D. (2005). Filling Up“: el problema de la independencia, preservando las intervenciones del maestro en lecciones con tareas de modelado exigentes. CERME 4 Actas del IV Congreso de la Sociedad Europea de Investigación en Educación Matemática, 1623-1633.

Blum, W., \& Niss, M. (1991). Resolución de problemas matemáticos aplicados, modelado, aplicaciones y vínculos con otras materias: estado, tendencias y problemas en la enseñanza de las matemáticas. studios educativos en matemáticas 22, 37-68.

Bosch, M., García, F., Gascón, J., \& Higueras, L. (2006). La modelización matemática y el problema de la articulación de la matemática escolar. Una propuesta desde la teoría antropológica de lo didáctico. Educación matemática, 3774.

Bressan, A., \& Gallego, M. (2011). La Educación Matemática Realista: Bases teóricas. III congreso nacional de matemática y problemáticas de la educación contemporánea. santa María, Argentina.

Brousseau, G. ((1997). Theory of Didactical Situations in Mathematics. Dordrecht: Dordrecht, Kluwer Academic Publishers. 
Buendía, G. (2013). La construcción social del conocimiento matemático escolar: un estudio socioepistemológico sobre la periodicidad de las funciones. Ediciones Díaz de Santos.

Bustos, A., Bustos, G., \& Novoa, Y. (2013). Propuesta de ambientes de aprendizaje para la promoción de la modelación matemática desde la perspectiva crítica. PROPOSAL LEARNING ENVIRONMENTS FOR PROMOTING MATHEMATICAL MODELING CRITICAL PERSPECTIV.

Camelo, F., Perilla, W., \& Mancera, G. (2016). Prácticas de modelación matemática desde una perspectiva socio crítica con estudiantes de grado undécimo. Revista Latinoamericana de Etnomatemática.

Cantoral, R. (2013). Teoría socioepistemológica de la matemática educativa. Estudios sobre construcción social del conocimiento (1a ed. Barcelona: Editorial Gedisa SA.

Cantoral, R., Montiel, G., \& Reyes-Gasperini, D. (2015). Análisis del discurso Matemático Escolar en los libros de texto, una mirada desde la Teoría Socioepestemológica. Avances de Investigación en Educación Matemática, 9-28.

Cárcamo, A., Gómez, J., \& Fortuny, J. (2015). Modelización matemática en la educación matemática realista: Una propuesta para contribuir a la construcción formal de álgebra lineal.

Chevallard, Y., \& Johsua, M.-A. (1985). La transposition didactique: du savoir savant au savoir enseigné. La Pensée sauvage.

Chevallard, Y., \& Sensevy, G. (2014). Anthropological approaches in mathematics education, French perspectives. Encyclopedia of mathematics education, 38-43.

Chevallard, Y., Bosch, M., \& Gascón, J. (1997). Estudiar matemáticas: el eslabón perdido entre la enseñanza y el aprendizaje. Barcelona: ICE Universitat de Barcelona.

Codero, F., Mena-Lorca, J., Huincahue, J., Mendoza, J., \& Pérez-Oxté, I. (2019). A category of modeling: the uses of mathematical knowledge in different scenarios and the learning of mathematics. Artículo en preparación.

Cordero, F. (2006). La modellazione e la rappresentazione grafica nell'insegnamento-apprendimento della matemática. La Matematica e la sua Didattica, 59-79.

Cordero, F. (2017). La matemática y lo matemático. Transversalidad y modelación: un programa socioepistemológico. Documento en preparación.

Espinosa, G., \& Buendía, G. (2012). Un Esquema Metodológico Para La Investigación Socioepistemológica: Ejemplos $\mathrm{E}$ Ilustraciones5. En A. Romo, \& A. Rosas, Metodología en Matemática Educativa: Visiones y reflexiones (págs. 55-82). México: Lectorum.

Fernández, J., \& Velázquez, A. (2007). Un ejemplo de la utilidad de los contextos en la matemática realista: los algoritmos de suma y resta por columnas. Uno: Revista de didáctica de las matematicas,, 95103.

Framework, A. (2003). Mathematics, Reading, Science, and Problem Solving Knowledge and Skills. París: OCDE. 
Gascón, J., Bosch, M., \& Ruiz, N. (2017). El problema del álgebra elemental en la teoría antropológica de lo didáctico.

Goffree, F. (2000). Principios y paradigmas de una educación matemática realista. En M. Gorgorió, J. Deulofeu, A. Bishop, K. Clements, P. Hilton, N. Balacheff, ... . M. Gorgorió, Matemáticas y educación : retos y cambios desde una perspectiva internacional (págs. 151-168). Barcelona: Graó.

Jacobini, O. (2004). modelagem matemática como instrumento de ação política na sala de aula. Tesis de Doctorado.

Kaiser, G., \& Sriraman, B. (2006). A global survey of international perspectives on modelling in mathematics education. The International Journal On Mathematics Education, 302-310.

King, R., Garrett, S., \& Coghill, G. (2005). On the use of qualitative reasoning to simulate and identify metabolic pathways. Bioinformatics.

Mancera, G., Camelo, F., Amaya, C., \& García, G. (2014). Aspectos políticos y críticos en las prácticas de modelación matemática escolar . Memorias Primer Encuentro Distrital de Educación Matemática .

Mancera, G., Camelo, F., Salazar, C., \& Valero, P. (2012). DISPOSICIONES, INTENCIONES Y ACCIONES: UNA VÍA PARA NEGOCIAR Y CONSTRUIR CAMPOS SEMÁNTICOS PARA LAS CLASES DE MATEMÁTICAS.

Montiel, G. (2011). Construcción de conocimiento trigonométrico: Un estudio Socioepistemológico. Ediciones Díaz de Santos.
Montiel, G., \& Buendía, G. .. (2013). Un esquema metodológico para la investigación socioepistemológica: ejemplos e ilustraciones. Metodología en matemática educativa: visiones $y$ reflexiones, 55-82.

Morales, A., \& Cordero, F. (2014). La graficaciónmodelación y la Serie de Taylor. Una socioepistemología del cálculo. Revista latinoamericana de investigación en matemática educativa, 319-345.

Munzón, N. (2010). La introducción del álgebra elemental y su desarrollo hacia la modelización funcional. Doctoral dissertation, Universitat Autònoma de Barcelona.

Panhuizen, M. (2008). Educación matemática en los Países Bajos: un recorrido guiado. Correo del maestro.

Pedreros, M. (2013). Modelización de situaciones de movimiento en un sistema algebraico computacional: una aproximación desde la teoría antropológica de lo didáctico $y$ el enfoque instrumental (Doctoral dissertation. Maestría en educación con énfasis en educación matemáticaUniversidad del Valle.

PERALTA, C. (2020). "LA CATEGORÍA DE MODELACIÓN Y EL CONCEPTO DE INTEGRAL DEFINIDA: UNA MIRADA SOCIOEPISTEMOLÓGICA." - UCMaule-Revista Académica de la Universidad Católica del Maule .

Perilla, W., Mancera, G., \& Camelo, F. (2016). La telefonía móvil en Colombia: Un ambiente de modelación matemática desde una perspectiva crítica. RECME: Revista Colombiana de Matemática Educativa. 
Perilla, W., Mancera, G., \& Camelo, F. (2016). Prácticas de modelación matemática desde una perspectiva socio crítica con estudiantes de grado undécimo. Revista Latinoamericana de Etnomatemática.

Rey, M. (2017). Modelización matemática: una mirada desde la teoría antropológica de lo didáctico. Montevideo: Actas del $7^{\circ}$ Congreso Uruguayo de Educación Matemática.

Rojas, N., Salazar, C., \& Romero, J. (2013). Lenguajes, preguntas y situaciones de variación: modelación y conocer reflexivo. Memorias VII Congreso Iberoamericano de Educación Matemática .

Salazar, C., Mancera, G., Camelo, F., \& Perilla, W. (2017). Una propuesta para el desarrollo de prácticas pedagógicas de modelación matemática en la perspectiva socio crítica. Encuentro Distrital deEducación Matemática EDEM-4 "Cultura, sociedad y escuela en la educación matemática del Distrito capital.

Skovsmose, O. (1994). Towards a philosophy of critical mathematics education. Boston: Kluwer Academic Publishers.

Skovsmose, O. (1999). Hacia una Filosofia de la Educación Matemática Crítica Bogotá. Bogotá: Empresa Docente.

Skovsmose, O. (2000). Escenarios de investigación. Revista EMA, 3-25.

Tellez, L., \& Cordero, F. (2010). Modelación Graficación, una categoría para la matemática escolar. Resultados de un estudio socioepistemológico. RELIME. Revista latinoamericana de investigación en matemática educativa, 319-333.
Treffers, A. (1987). "Three Dimensions. A model of goal and theory description in mathematics instruction:The Wiskobas project”. Dordrecht (Holanda): Springer.

Valero, P. (2002). Consideraciones sobre el contexto y la educación matemática para la democracia. Revista Teorica e de Investigaça.

Vanegas, J., \& Henao, S. (2013). Educación Matemática Realista: la modelación matemática en la producción y uso de modelos cuadráticos.

Villa, J. (2007). La modelación como proceso en el aula de matemáticas: un marco de referencia y un ejemplo. Tecno Lógicas, 19.

Zamora, P. (2013). La contextualización de las matemáticas. Almeria : Universidad de Almeria . 